\title{
The Numerical Investigation of Fractional-Order Zakharov-Kuznetsov Equations
}

\author{
Pongsakorn Sunthrayuth $\left(D,{ }^{1}\right.$ Farman Ali $\left(D,{ }^{2}\right.$ A. A. Alderremy, ${ }^{3}$ Rasool Shah $\left(D,{ }^{4}\right.$ \\ Shaban Aly, ${ }^{5}$ Y. S. Hamed $\left(\mathbb{D},{ }^{6}\right.$ and Jeevan Katle ${ }^{7}{ }^{7}$ \\ ${ }^{1}$ Department of Mathematics and Computer Science, Faculty of Science and Technology, \\ Rajamangala, University of Technology Thanyaburi (RMUTT), Pathumthani 12110, Thailand \\ ${ }^{2}$ Department of Software, Sejong Unversity, Seoul 05006, Republic of Korea \\ ${ }^{3}$ Department of Mathematics, Faculty of Science, King Khalid University, Abha 61413, Saudi Arabia \\ ${ }^{4}$ Department of Mathematics, Abdul Wali Khan University, Mardan 23200, Pakistan \\ ${ }^{5}$ Department of Mathematics, Faculty of Science, AL-Azhar University, Assiut, Egypt \\ ${ }^{6}$ Department of Mathematics and Statistics, College of Science, Taif University, P O. Box 11099, Taif 21944, Saudi Arabia \\ ${ }^{7}$ Central Department of Mathematics, Tribhuvan University, Kritipur, Nepal
}

Correspondence should be addressed to Jeevan Katle; jeevan.kafle@cdmath.tu.edu.np

Received 1 May 2021; Revised 19 May 2021; Accepted 2 November 2021; Published 27 November 2021

Academic Editor: Dan Selişteanu

Copyright (C) 2021 Pongsakorn Sunthrayuth et al. This is an open access article distributed under the Creative Commons Attribution License, which permits unrestricted use, distribution, and reproduction in any medium, provided the original work is properly cited.

In this article, a modified method called the Elzaki decomposition method has been applied to analyze time-fractional Zakharov-Kuznetsov equations. In this method, the Adomian decomposition technique and Elzaki transformation are combined. Two problems are investigated to show and validate the efficiency of the suggested method. It is also shown that the solutions achieved from the current producer are in good contact with the exact solutions to show with the help of graphs and table. It is observed that the suggested technique is to be reliable, efficient, and straightforward to implement for many related models of engineering and science.

\section{Introduction}

Nonlinear fractional partial differential equations play important role in demonstrating different physical appearances identified with solid-state physics, fluid mechanics, chemical kinetics, population dynamics, plasma physics, nonlinear optics, protein chemistry, soliton theory, etc. These nonlinear problems, just as their scientific arrangements, are of tremendous enthusiasm for suitable subjects. In many above-discussed science and engineering areas, the nonlinear problems perform a key factor in many phenomena. Differential equations demonstrate several frameworks and the majority of them are nonlinear [1-4].

The Zakharov-Kuznetsov (ZK) equation is an extremely appealing model equation for investigating vortices in geophysical streams. The ZK problems show up in numerous regions of material science, implemented arithmetic, and designing. Specifically, it appears in the territory of quantum physics [5-9]. The ZK problems administer the conduct of feebly nonlinear particle acoustic plasma waves, including cold particles and hot isothermal electrons within sight of a smooth magnetic field $[10,11]$. Solitary wave arrangements were produced by determining the nondirect higher order of broadened $\mathrm{KdV}$ conditions for the free surface removal [12]. By utilizing fractional strategy, the precise expository structures of some nonlinear advancement equations in numerical material science, to be specific, space timefractional Zakharov-Kuznetsov and modified Zakharov-Kuznetsov equations, were obtained [13]. It has been investigated in the past decades by many with the 
techniques such as new iterative Sumudu transform method [14], homotopy perturbation transform method [15], extended direct algebraic method [16], natural decomposition method, and q-homotopy analysis transform method [17].

In the last three decades, fractional differential conditions have picked up importance and ubiquity, mostly since its exhibited uses in various fields of material science and design. Numerous significant phenomena in electromagnetics, acoustics, viscoelasticity, electrochemistry and material science, likelihood and measurements, electrochemistry of erosion, concoction physical science, and sign preparation are depicted in fractional differential equations [18-23]. Consequently, special consideration has been given to discover solutions of fractional differential equations.

The investigation of these equations and their solutions has extraordinary enthusiasm for numerous specialists because of its different applications. To refer to a couple, Wazwaz [24] used the Adomian disintegration strategy as a dependable method for treating Schrodinger conditions. In Wazwaz [25], the variational emphasis technique was utilized to obtain specific solutions for both linear and nonlinear Schrodinger equations. Additionally, Shah et al. [26] utilized He's recurrence definition as a technique to look for Schrodinger equations arrangements. The arrangements decided to end up being in good concurrence with the outcomes decided in $[24,25]$. Notwithstanding, we mean to couple the Elzaki transform built up as late by Elzaki [27] with the commended technique for the 80th Adomian decay strategy $[28,29]$. Recently, many researchers obtained the results of FPDEs; interested readers can see [30-36].

In this present work, the Elzaki decomposition technique is applied to investigate the result of the fractional-order ZK equation. The fractional derivatives are defined by the Caputo operator. The result of the given problems shows the validity of the suggested method. The solutions of the suggested technique are analyzed and shown with the help of the table and figures. Applying the current method, the results of time-fractional equations and integral-order equations are investigated. The given method is very helpful in solving other fractional-order of PDEs.

\section{Basic Definitions}

2.1. Definition. The fractional-order Riemann-Liouville $\rho>0$, of a function $f \in C_{1}, \rho \geq-1$, is given as [27]

$$
\begin{aligned}
& J^{\rho} h(\xi)=\frac{1}{\Gamma(\rho)} \int_{0}^{\xi}(\xi-1)^{\rho-1} h(\eta) \partial \eta, \quad \rho, \xi>0, \\
& J^{\rho} h(\xi)=h(\xi) .
\end{aligned}
$$

The operator of some properties:

For $h \in C_{1}, \rho \geq-1, \rho, \beta \geq 0$, and $\rho>-1$,

$$
\begin{aligned}
J^{\rho} J^{\beta} h(\xi) & =J^{\rho+\beta} h(\xi), \\
J^{\rho} J^{\beta} h(\xi) & =J^{\rho} J^{\beta} h(\xi), \\
J^{\beta} \xi^{\rho} & =\frac{\Gamma(\rho+1)}{(\beta+\rho+1)} \xi^{\beta+\rho} .
\end{aligned}
$$

2.2. Lemma. If $1-1<\rho \leq 1,1 \in \mathrm{N}$ and $h \in C_{1}, \rho \geq-1$, then $D^{\rho} J^{\rho} h(\xi)=h(\xi)[18-20]$,

$$
D^{\rho} J^{\rho} h(\xi)=h(\xi)-\sum_{1=0}^{m-1} h^{(1)}(0) \frac{\xi^{1}}{1 !}, \quad \xi>0 .
$$

The basic theory of the Elzaki transformation:

A new transform called the Elzaki transform defines the function exponential order that we found in the set $A$, define by [27]

$$
A=\left\{h(\mathfrak{I}): \sum\left|M, k_{1}, k_{2}>0,\right| h(\Im) \mid<M e^{|\Im| ⿰ \mid k_{1}}, \quad \text { if }(\Im) \in(-1)^{1} \times[0, \infty)\right.
$$


The finite number $M$ must be constant, $k_{1}$ and $k_{2}$ of infinite or finite, for a specified function in the set. The Elzaki transformation is defined throughout the following integral problem:

$$
\mathrm{E}[h(\mathfrak{I})]=T(s)=s \int_{0}^{\infty} h(\mathfrak{I}) e^{-\Im / s} \mathrm{~d} \mathfrak{I}, \quad \mathfrak{I} \geq 0, k_{1} \leq s \leq k_{2} .
$$

We can obtain the next solution from the explanation and the basic investigation

$$
\begin{aligned}
E\left[\mathfrak{I}^{n}\right] & =m ! s^{m+2}, \\
E\left[h^{\prime}(\mathfrak{I})\right] & =\frac{T(s)}{s}-s h(0), \\
E\left[h^{\prime \prime}(\mathfrak{I})\right] & =\frac{T(s)}{s^{2}}-h(0)-s h^{\prime}(0), \\
E\left[h^{(m)}(\mathfrak{I})\right] & =\frac{T(s)}{s^{m}}-\sum_{k=0}^{n-1} s^{2-m+k} h^{(k)}(0) .
\end{aligned}
$$

2.3. Theorem. The Elzaki Riemann-Liouville transform of the derivative can be defined as given if $T(s)$ is the Elzaki transformation of (I) [27]:

$$
E\left[D^{\rho} h(\Im)\right]=s^{-\rho}\left[T(s)-\sum_{k=1}^{m}\left\{D^{\rho-k} h(0)\right\}\right], \quad-1<m-1 \leq \rho<m .
$$

proof. Taking the Laplace transformation of $h^{\prime}(\mathfrak{J})=d / \mathrm{d} \Im h(\Im)$, we have

$$
\begin{aligned}
L\left[D^{\rho} h(\Im)\right] & =s^{\rho} T(s)-\sum_{k=0}^{m-1} s^{k}\left[D^{\rho-k-1} h(0)\right] \\
& =s^{\rho} T(s)-\sum_{k=0}^{m-1} s^{k-1}\left[D^{\rho-k} h(0)\right]=s^{\rho} T(s)-\sum_{k=0}^{m-1} s^{k-2}\left[D^{\rho-k} h(0)\right] \\
& =s^{\rho} T(s)-\sum_{k=0}^{m-1} \frac{1}{s^{-k+2}}\left[D^{\rho-k} h(0)\right]=s^{\rho} T(s)-\sum_{k=0}^{m-1} \frac{1}{s^{\rho-k+2-\rho}}\left[D^{\rho-k} h(0)\right] \\
& =s^{\rho} T(s)-\sum_{k=0}^{m-1} s^{\rho} \frac{1}{s^{\rho-k+2}}\left[D^{\rho-k} h(0)\right] \\
L\left[D^{\rho} h(\Im)\right] & =s^{\rho}\left[T(s)-\sum_{k=0}^{m-1}\left(\frac{1}{s}\right)^{\rho-k+2}\left[D^{\rho-k} h(0)\right]\right] .
\end{aligned}
$$
is

Therefore, the fractional-order Elzaki transform of $h(\mathfrak{J})$

$$
E\left[D^{\rho} h(\mathfrak{\Im})\right]=s^{-\rho}\left[T(s)-\sum_{k=0}^{m}(s)^{\rho-k+2}\left[D^{\rho-k} h(0)\right]\right] .
$$

2.4. Definition. Using Theorem 1, the fractional Caputo ET is provided as [18-20]

$$
\mathrm{E}\left[D_{\Im}^{\rho} g(\mathfrak{I})\right]=s^{\rho} \mathrm{E}[g(\mathfrak{I})]-\sum_{k=0}^{1-1} s^{2-\rho+k} g^{(k)}(0), \quad \text { where } 1-1<\rho<1 .
$$




\section{The General Implementation of Elzaki Decomposition Technique}

In this section, we present the Elzaki decomposition technique producer for fractional partial differential equation. $D^{\rho} \Psi(\xi, \mathfrak{\Im})+L \Psi(\xi, \mathfrak{\Im})+N \Psi(\xi, \mathfrak{\Im})=q(\xi, \mathfrak{\Im}), \quad \xi, \mathfrak{\Im} \geq 0,1-1<\rho<1$,

$$
\Psi(\xi, 0)=k(\xi)
$$

Applying the Elzaki transform to equation (11), we get $E\left[D^{\rho} \Psi(\xi, \mathfrak{\Im})\right]+E[L \Psi(\xi, \mathfrak{\Im})+N \Psi(\xi, \mathfrak{\Im})]=E[q(\xi, \mathfrak{\Im})]$.

Using the Elzaki transform differentiation property,

and the initial condition is

$$
\begin{aligned}
\frac{1}{s^{\rho}} E[\Psi(\xi, \mathfrak{I})]-s^{2-\rho} \Psi(\xi, 0) & =E[q(\xi, \mathfrak{\Im})]-E[L \Psi(\xi, \mathfrak{\Im})+N \Psi(\xi, \mathfrak{\Im})] \\
E[\Psi(\xi, \mathfrak{\Im})] & =s^{2} \Psi(\xi, 0)+s^{\rho} E[q(\xi, \mathfrak{\Im})]-s^{\rho} E[L \Psi(\xi, \mathfrak{\Im})+N \Psi(\xi, \mathfrak{J})] .
\end{aligned}
$$

Now, $\Psi(\xi, 0)=k(\xi)$ and hence

$E[\Psi(\xi, \Im)]=s^{2} k(\xi)+s^{\rho} E[q(\xi, \Im)]-s^{\rho} E[L \Psi(\xi, \Im)+N \Psi(\xi, \Im)]$,

where $\Psi(\xi, \mathfrak{I})$ is defined as

$$
\Psi(\xi, \mathfrak{\Im})=\sum_{1=0}^{\infty} \Psi_{1}(\xi, \mathfrak{J})
$$

The nonlinearity of Adomian polynomials terms $N$ is defined as

$$
N \Psi(\xi, \mathfrak{\Im})=\sum_{1=0}^{\infty} A_{1}
$$

$$
A_{1}=\frac{1}{1 !}\left[\frac{d^{1}}{\mathrm{~d} \lambda^{1}}\left[N \sum_{1=0}^{\infty}\left(\lambda^{1} \Psi_{1}\right)\right]\right]_{\lambda=0}, \quad 1=0,1,2, \ldots
$$

Putting equation (16) and (17) into (15), we have

$$
E\left[\sum_{1=0}^{\infty} \Psi_{1}(\xi, \mathfrak{\Im})\right]=s^{2} k(\xi)+s^{\rho} E[q(\xi, \mathfrak{\Im})]-s^{\rho} E\left[L \sum_{1=0}^{\infty} \Psi_{1}(\xi, \mathfrak{J})+\sum_{1=0}^{\infty} A_{1}\right]
$$

Now using EDM, we have

$$
E\left[\Psi_{0}(\xi, \Im)\right]=s^{2} k(\xi)+s^{\rho} E[q(\xi, \Im)] .
$$

Generally, we can write

$$
E\left[\Psi_{1+1}(\xi, \mathfrak{J})\right]=-s^{\rho} E\left[L \Psi_{1}(\xi, \mathfrak{J})+A_{1}\right], \quad 1 \geq 1 .
$$
have

Taking the inverse Elzaki transform of equation (21), we

$$
\begin{aligned}
& \Psi_{0}(\xi, \mathfrak{J})=k(\xi)+E^{-1}\left[s^{\rho} E[q(\xi, \mathfrak{\Im})]\right], \\
& \Psi_{1+1}(\xi, \mathfrak{I})=-E^{-1}\left[s^{\rho} E\left[L \Psi_{1}(\xi, \mathfrak{I})+A_{1}\right]\right] \text {. }
\end{aligned}
$$

\section{Main Results}

Example 1. Consider the two-dimensional Zakharov-Kuznetsov equation as

$$
D_{\mathfrak{J}}^{\rho} \Psi+\left(\Psi^{2}\right)_{\xi}+\frac{1}{8}\left(\Psi^{2}\right)_{\xi \xi \xi}+\frac{1}{8}\left(\Psi^{2}\right)_{\xi \zeta \zeta}=0, \quad 0<\rho \leq 1,
$$

and the initial condition is

$$
\Psi(\xi, \zeta, 0)=\frac{4}{3} \eta \sinh ^{2}(\xi+\zeta),
$$

where $\eta$ is an arbitrary constant.

Taking Elzaki transform of equation (23), we have

$$
\begin{aligned}
E\left[\frac{\partial^{\rho} \Psi}{\partial \mathfrak{J}^{\rho}}\right] & =E\left[-\left(\Psi^{2}\right)_{\xi}-\frac{1}{8}\left(\Psi^{2}\right)_{\xi \xi \xi}-\frac{1}{8}\left(\Psi^{2}\right)_{\xi \zeta \zeta}\right], \\
\frac{1}{s^{\rho}} E[\Psi(\xi, \zeta, \mathfrak{J})]-s^{2-\rho}[\Psi(\xi, \zeta, 0)] & =E\left[-\left(\Psi^{2}\right)_{\xi}-\frac{1}{8}\left(\Psi^{2}\right)_{\xi \xi \xi}-\frac{1}{8}\left(\Psi^{2}\right)_{\xi \zeta \zeta}\right] .
\end{aligned}
$$


Applying the inverse Elzaki transform, we have

$$
\begin{aligned}
& \Psi(\xi, \zeta, \mathfrak{\Im})=E^{-1}\left(s^{2} \Psi(\xi, \zeta, 0)\right)+E^{-1}\left[s^{\rho} E\left[-\left(\Psi^{2}\right)_{\xi}-\frac{1}{8}\left(\Psi^{2}\right)_{\xi \xi \xi}-\frac{1}{8}\left(\Psi^{2}\right)_{\xi \zeta \zeta}\right]\right], \\
& \Psi(\xi, \zeta, \mathfrak{J})=\frac{4}{3} \eta \sinh ^{2}(\xi+\zeta)+E^{-1}\left[s^{\rho} E\left[-\left(\Psi^{2}\right)_{\xi}-\frac{1}{8}\left(\Psi^{2}\right)_{\xi \xi \xi}-\frac{1}{8}\left(\Psi^{2}\right)_{\xi \zeta \zeta}\right]\right] .
\end{aligned}
$$

Using ADM procedure, we get

$$
\sum_{1=0}^{\infty} \Psi_{1}(\xi, \zeta, \mathfrak{\Im})=\frac{4}{3} \eta \sinh ^{2}(\xi+\zeta)+E^{-1}\left[s^{\rho} E\left[-N(\Psi)_{\xi}-\frac{1}{8} N(\Psi)_{\xi \xi \xi}-\frac{1}{8} N(\Psi)_{\zeta \zeta \xi}\right]\right]
$$

where the nonlinear terms can be defined by Adomian polynomials in the above equations.

$$
N(\Psi)=\Psi^{2}=\sum_{1=0}^{\infty} \mathscr{A}_{1}(\Psi)
$$

$$
\begin{aligned}
\mathscr{A}_{0} & =\Psi_{0}^{2} \\
\mathscr{A}_{1} & =2 \Psi_{0} \Psi_{1} \\
\mathscr{A}_{2} & =2 \Psi_{0} \Psi_{2}+\Psi_{1}^{2} \\
\Psi_{0}(\xi, \zeta, \mathfrak{I}) & =\frac{4}{3} \eta \sinh ^{2}(\xi+\zeta) \\
\Psi_{1+1}(\xi, \zeta, \mathfrak{I}) & =E^{-1}\left[s^{\rho} E\left[-\left(\sum_{1=0}^{\infty} \mathscr{A}_{1}(\Psi)\right)_{\xi}-\frac{1}{8}\left(\sum_{1=0}^{\infty} \mathscr{A}_{1}(\Psi)\right)_{\xi \xi \xi}-\frac{1}{8}\left(\sum_{1=0}^{\infty} \mathscr{A}_{1}(\Psi)\right)_{\zeta \zeta \xi}\right]\right]
\end{aligned}
$$

for $1=0,1,2, \ldots$,

$$
\begin{aligned}
& \Psi_{1}(\xi, \zeta, \Im)=E^{-1}\left[s^{\rho} E\left[-\left(\Psi_{0}^{2}\right)_{\xi}-\frac{1}{8}\left(\Psi_{0}^{2}\right)_{\xi \xi \xi}-\frac{1}{8}\left(\Psi_{0}^{2}\right)_{\xi \zeta \zeta}\right]\right], \\
& \Psi_{1}(\xi, \zeta, \Im)=\left(-\frac{224}{9} \eta^{2} \sinh ^{3}(\xi+\zeta) \cosh (\xi+\zeta)-\frac{32}{3} \eta^{2} \sinh (\xi+\zeta) \cosh ^{3}(\xi+\zeta)\right) E^{-1}\left(s^{\rho+2}\right) \\
& \Psi_{1}(\xi, \zeta, \Im)=\left(-\frac{224}{9} \eta^{2} \sinh ^{3}(\xi+\zeta) \cosh (\xi+\zeta)-\frac{32}{3} \eta^{2} \sinh (\xi+\zeta) \cosh ^{3}(\xi+\zeta)\right) \frac{\mathfrak{J}^{\rho}}{\Gamma(\rho+1)}
\end{aligned}
$$


The few terms of the given methods are

$$
\begin{aligned}
& \Psi_{2}(\xi, \zeta, \mathfrak{I})=E^{-1}\left[s^{\rho} E\left[-\left(2 \Psi_{0} \Psi_{1}\right)_{\xi}-\frac{1}{8}\left(2 \Psi_{0} \Psi_{1}\right)_{\xi \xi \xi}-\frac{1}{8}\left(2 \Psi_{0} \Psi_{1}\right)_{\xi \zeta \zeta}\right]\right] \\
& \Psi_{2}(\xi, \zeta, \mathfrak{\Im})=\frac{128}{27} \eta^{3}\left(1200 \cosh ^{6}(\xi+\zeta)-2080 \cosh ^{4}(\xi+\zeta)+968 \cosh ^{2}(\xi+\zeta)-79\right) \frac{\mathfrak{J}^{2 \rho}}{\Gamma(2 \rho+1)} \\
& \Psi_{3}(\xi, \zeta, \mathfrak{\Im})=E^{-1}\left[s^{\rho} E\left[-\left(2 \Psi_{0} \Psi_{2}+\Psi_{1}^{2}\right)_{\xi}-\frac{1}{8}\left(2 \Psi_{0} \Psi_{2}+\Psi_{1}^{2}\right)_{\xi \xi \xi}-\frac{1}{8}\left(2 \Psi_{0} \Psi_{2}+\Psi_{1}^{2}\right)_{\xi \zeta \zeta}\right]\right] \\
& \Psi_{3}(\xi, \zeta, \mathfrak{\Im})=-\frac{2048}{81} \eta^{4} \sinh (\xi+\zeta) \cosh (\xi+\zeta)\left[88400 \cosh ^{6}(\xi+\zeta)-160200\right. \\
& \left.\cosh ^{4}(\xi+\zeta)+85170 \cosh ^{2}(\xi+\zeta)-11903\right] \frac{\mathfrak{J}^{3 \rho}}{\Gamma(3 \rho+1)}
\end{aligned}
$$

The EDM result is

$$
\begin{aligned}
\Psi(\xi, \zeta, \mathfrak{I})= & \Psi_{0}(\xi, \zeta, \mathfrak{I})+\Psi_{1}(\xi, \zeta, \mathfrak{I})+\Psi_{2}(\xi, \zeta, \mathfrak{I})+\Psi_{3}(\xi, \zeta, \mathfrak{I})+\cdots \\
\Psi(\xi, \zeta, \mathfrak{I})= & \frac{4}{3} \eta \sinh (\xi+\zeta)-\left(\frac{224}{9} \eta^{2} \sinh ^{3}(\xi+\zeta) \cosh (\xi+\zeta)+\frac{32}{3} \eta^{2} \sinh (\xi+\zeta) \cosh ^{3}(\xi+\zeta)\right) \frac{\mathfrak{J}^{\rho}}{\Gamma(\rho+1)} \\
& +\frac{128}{27} \eta^{3}\left(1200 \cosh ^{6}(\xi+\zeta)-2080 \cosh ^{4}(\xi+\zeta)+968 \cosh ^{2}(\xi+\zeta)-79\right) \frac{\mathfrak{J}^{2 \rho}}{\Gamma(2 \rho+1)}-\frac{2048}{81} \eta^{4} \sinh (\xi+\zeta) \\
& \cosh (\xi+\zeta)\left[88400 \cosh ^{6}(\xi+\zeta)-160200 \cosh ^{4}(\xi+\zeta)+85170 \cosh ^{2}(\xi+\zeta)-11903\right] \frac{\mathfrak{J}^{3 \rho}}{\Gamma(3 \rho+1)}+\cdots
\end{aligned}
$$

For $\rho=1$, we have

$$
\Psi(\xi, \Im)=\frac{4}{3} \eta \sinh ^{2}(\xi+\zeta-\eta \Im)
$$

In Figure 1, the exact and the EDM solutions of problem 1 at $\rho=1$ are shown by Figures 1 (a) and 1(b), respectively. From the given figures, it can be seen that both the EDM and exact results are in close contact with each other. Also, in Figures 1(c) and 1(d), the EDM solutions of problem 1 are investigated at different fractional-order $\rho=0.8$ and 0.6 . It is analyzed that time-fractional problem results are convergent to an integer order effect as time-fractional analysis to integer order. In Figure 2, the first graph shows the two dimensions of exact and analytical solutions with respect to $\xi$ and $\mathfrak{I}$ and second one shows the different fractional-order graph with respect to $\xi$ and $\mathfrak{\Im}$. Table 1 shows the different fractional-order absolute error.

Example 2. Consider the three-dimensional Zakharov-Kuznetsov equation as

$$
D_{\Im}^{\rho} \Psi+\left(\Psi^{3}\right)_{\xi}+2\left(\Psi^{3}\right)_{\xi \xi \xi}+2\left(\Psi^{3}\right)_{\xi \zeta \zeta}=0, \quad 0<\rho \leq 1,
$$

and the initial condition is

$$
\Psi(\xi, \zeta, 0)=\frac{3}{2} \eta \sinh \left[\frac{1}{6}(\xi+\zeta)\right]
$$

where $\eta$ is an arbitrary constant.

Taking Elzaki transform of equation (34), we have

$$
\begin{array}{r}
E\left[\frac{\partial^{\rho} \Psi}{\partial \mathfrak{\Im}^{\rho}}\right]=E\left[-\left(\Psi^{3}\right)_{\xi}-2\left(\Psi^{3}\right)_{\xi \xi \xi}-2\left(\Psi^{3}\right)_{\xi \zeta \zeta}\right], \\
s^{\rho} E[\Psi(\xi, \zeta, \Im)]-s^{2-\rho}[\Psi(\xi, \zeta, 0)]=E\left[-\left(\Psi^{3}\right)_{\xi}-2\left(\Psi^{3}\right)_{\xi \xi \xi}-2\left(\Psi^{3}\right)_{\xi \zeta \zeta}\right] .
\end{array}
$$




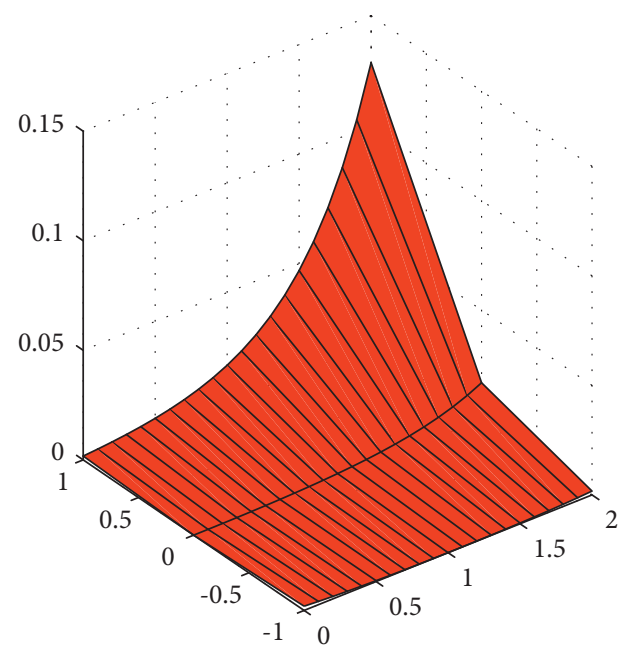

(a)

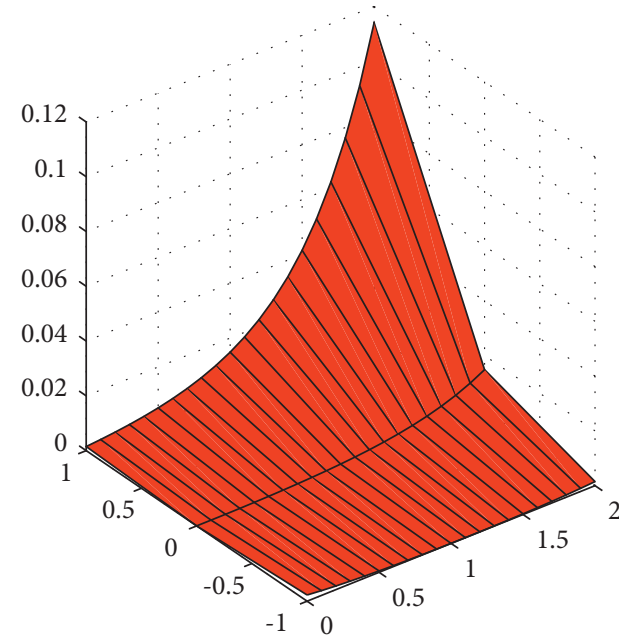

(c)
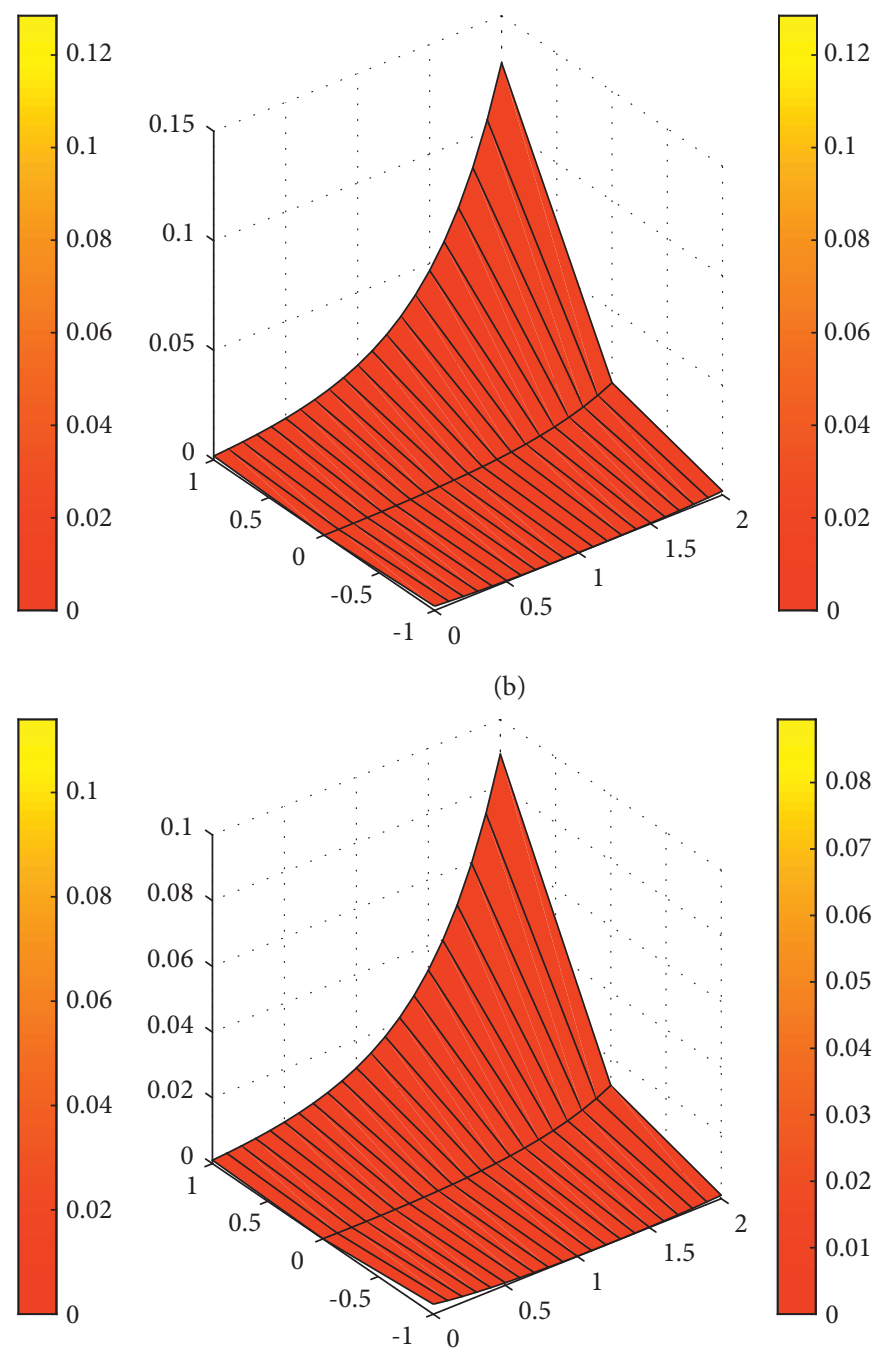

(d)

Figure 1: (a) The exact solution figure of $\Psi(\xi, \mathfrak{\Im})$ of Example 1. (b) The EDM solution figure of $\Psi(\xi, \mathfrak{I})$ of Example 1. (c) The graph EDM result of $\Psi(\xi, \mathfrak{\Im})$ at $\rho=0.8$ problem 1 . (d) The graph $\operatorname{EDM}$ result of $\Psi(\xi, \mathfrak{\Im})$ at $\rho=0.6$ problem 1 .

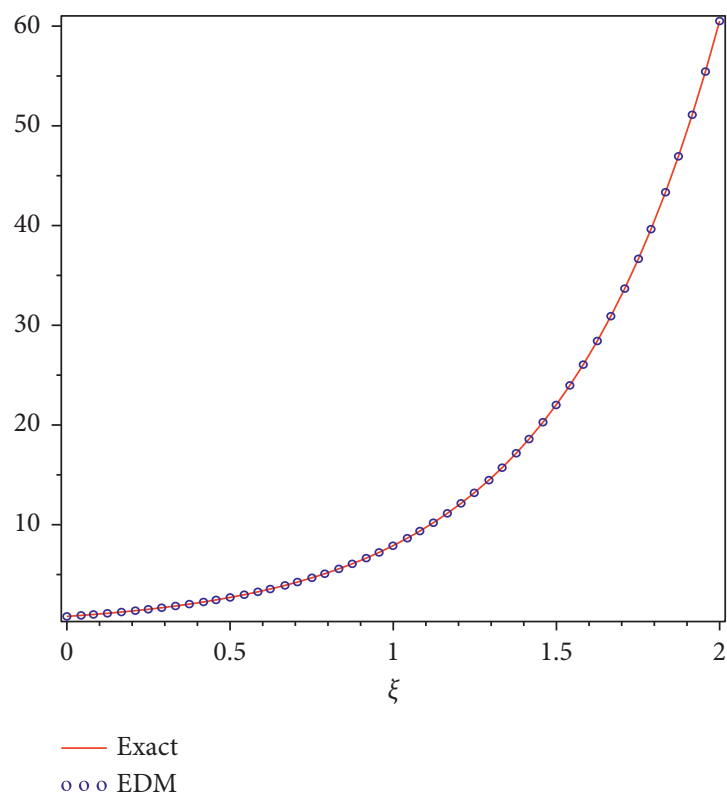

(a)

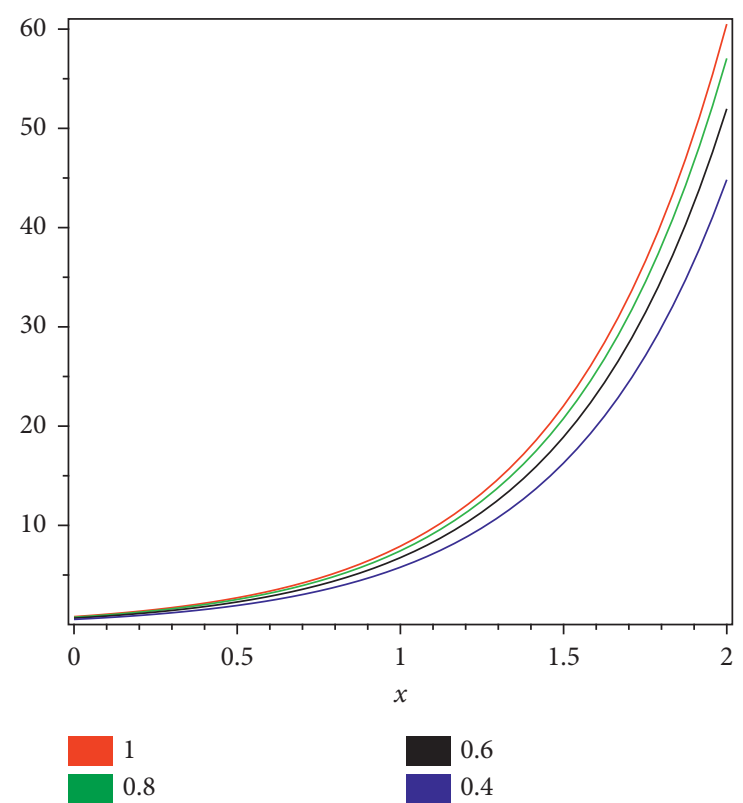

(b)

Figure 2: Continued. 


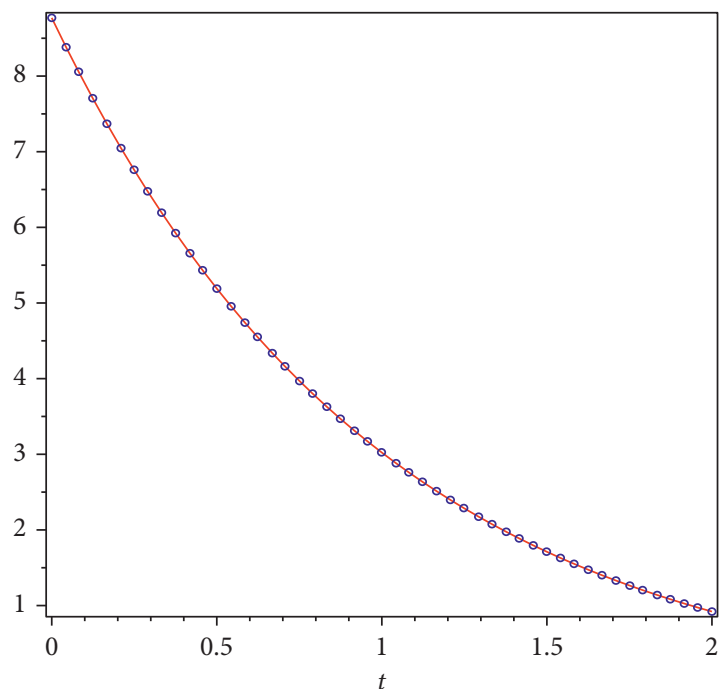

(c)

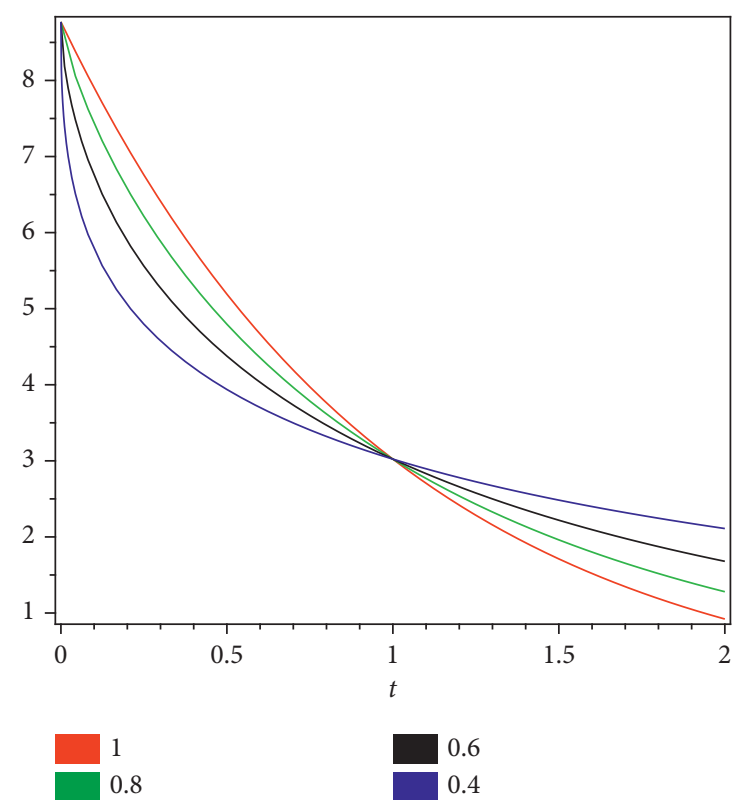

(d)

Figure 2: (a) The exact and EDM solution figure of $\Psi(\xi, \mathfrak{\Im})$ of Example 1. (b) The EDM solution figure of different fractional-order of $\rho$ at $\Psi(\xi, \mathfrak{I})$ of Example 1. (c) The exact and EDM solution figure of $\Psi(\xi, \mathfrak{I})$ with respect to $\mathfrak{I}$ Example 1 . (d) The EDM solution figure of different fractional-order of $\rho$ with respect to $\mathfrak{J}$ of Example 1 .

TABLE 1: EDM result for a different value of $\rho$ when $\eta=0.001$ and absolute error of Example 1.

\begin{tabular}{|c|c|c|c|c|c|c|c|}
\hline \multicolumn{8}{|c|}{ EDM } \\
\hline$\xi$ & $\zeta$ & $\mathfrak{I}$ & $\rho=0.6$ & $\rho=0.8$ & $\operatorname{EDM}(\rho=1)$ & Exact (E) & $\operatorname{AE}(\mathrm{E})(\rho=1)$ \\
\hline \multirow{3}{*}{0.2} & \multirow{3}{*}{0.2} & 0.1 & $6.347977 E-5$ & $6.378034 E-5$ & $4.461135 E-5$ & $4.488458-5$ & $2.7323-7$ \\
\hline & & 0.3 & $6.374898 E-5$ & $6.397867 E-5$ & $4.441928 E-5$ & 4.499518-5 & $5.76800-7$ \\
\hline & & 0.5 & $6.329478 E-5$ & $6.358477 E-5$ & $4.393236 E-5$ & $4.488588-5$ & 8.92644-7 \\
\hline \multirow{3}{*}{0.5} & \multirow{3}{*}{0.5} & 0.1 & $3.961810 E-3$ & $3.992936 E-3$ & $4.124545 E-3$ & $4.148347-3$ & $2.3802-5$ \\
\hline & & 0.3 & $3.918638 E-3$ & $3.937942 E-3$ & $2.767287 E-3$ & $2.868188-3$ & $1.00902-4$ \\
\hline & & 0.5 & $2.851320 E-3$ & $3.986888 E-3$ & $3.014248 E-3$ & $3.144685-3$ & $1.31437-4$ \\
\hline \multirow{3}{*}{1.0} & \multirow{3}{*}{1.0} & 0.1 & $2.651681 E-2$ & $2.755798 E-2$ & $2.78556 E-02$ & $2.864629-2$ & $7.9069-4$ \\
\hline & & 0.3 & $2.147458 E-2$ & $2.554775 E-2$ & $2.69884 E-02$ & $2.863881-2$ & $1.65041-3$ \\
\hline & & 0.5 & $3.686988 E-3$ & $3.286775 E-2$ & $2.55842 E-02$ & 2.861074-2 & $3.02654-3$ \\
\hline
\end{tabular}

Using inverse Elzaki transformation,

$$
\begin{aligned}
& \Psi(\xi, \zeta, \mathfrak{\Im})=E^{-1}\left[s^{2} \Psi(\xi, \zeta, 0)+s^{\rho} E\left[-\left(\Psi^{3}\right)_{\xi}-2\left(\Psi^{3}\right)_{\xi \xi \xi}-2\left(\Psi^{3}\right)_{\xi \zeta \zeta}\right]\right] \\
& \Psi(\xi, \zeta, \mathfrak{\Im})=\frac{3}{2} \eta \sinh \left[\frac{1}{6}(\xi+\zeta)\right]+E^{-1}\left[s^{\rho} E\left[-\left(\Psi^{3}\right)_{\xi}-2\left(\Psi^{3}\right)_{\xi \xi \xi}-2\left(\Psi^{3}\right)_{\xi \zeta \zeta}\right]\right] .
\end{aligned}
$$

Applying the procedure of ADM, we get

$$
\sum_{1=0}^{\infty} \Psi_{1}(\xi, \zeta, \Im)=\frac{3}{2} \eta \sinh \left[\frac{1}{6}(\xi+\zeta) t\right]+E^{-1}\left[s^{\rho} E\left[-N(\Psi)_{\xi}-2 N(\Psi)_{\xi \xi \xi}-2 N(\Psi)_{\xi \zeta \zeta}\right]\right]
$$


where the nonlinear terms can be defined by Adomian polynomials in the above equations.

$$
N(\Psi)=\Psi^{3}=\sum_{1=0}^{\infty} \mathscr{B}_{1}(\Psi) .
$$

$$
\begin{aligned}
\mathscr{B}_{0} & =\Psi_{0}^{3}, \\
\mathscr{B}_{1} & =3 \Psi_{0}^{2} \Psi_{1}, \\
\mathscr{B}_{2} & =3 \Psi_{0}^{2} \Psi_{2}+3 \Psi_{0}^{2} \Psi_{1}^{2}, \\
\Psi_{0}(\xi, \zeta, \Im) & =\frac{3}{2} \eta \sinh \left[\frac{1}{6}(\xi+\zeta)\right], \\
\Psi_{1+1}(\xi, \zeta, \mathfrak{J}) & =E^{-1}\left[s^{\rho} E\left[-\sum_{1=0}^{\infty} \mathscr{B}_{1}(\Psi)_{\xi}-2 \sum_{1=0}^{\infty} \mathscr{B}_{1}(\Psi)_{\xi \xi \xi}-2 \sum_{1=0}^{\infty} \mathscr{B}_{1}(\Psi)_{\xi \zeta \zeta}\right]\right],
\end{aligned}
$$

for $1=0,1,2, \ldots$,

$$
\begin{aligned}
& \Psi_{1}(\xi, \zeta, \mathfrak{\Im})=E^{-1}\left[s^{\rho} E\left[-\left(\Psi_{0}^{3}\right)_{\xi}-2\left(\Psi_{0}^{3}\right)_{\xi \xi \xi}-2\left(\Psi_{0}^{3}\right)_{\xi \zeta \zeta}\right]\right] \\
& \Psi_{1}(\xi, \zeta, \mathfrak{\Im})=-\left[3 \eta^{3} \sinh ^{2}\left[\frac{1}{6}(\xi+\zeta)\right] \cosh \left[\frac{1}{6}(\xi+\zeta)\right]+\frac{3}{8} \eta^{3} \cosh ^{3}\left[\frac{1}{6}(\xi+\zeta)\right]\right] E^{-1}\left(\frac{1}{s^{\rho+2}}\right) \\
& \Psi_{1}(\xi, \zeta, \mathfrak{I})=-\left[3 \eta^{3} \sinh ^{2}\left[\frac{1}{6}(\xi+\zeta)\right] \cosh \left[\frac{1}{6}(\xi+\zeta)\right]+\frac{3}{8} \eta^{3} \cosh ^{3}\left[\frac{1}{6}(\xi+\zeta)\right]\right] \frac{\mathfrak{J}^{\rho}}{\Gamma(\rho+1)}
\end{aligned}
$$

The subsequent terms are

$$
\begin{aligned}
\Psi_{2}(\xi, \zeta, \mathfrak{I})= & E^{-1}\left[s^{\rho} E\left[-\left(3 \Psi_{0}^{2} \Psi_{1}\right)_{\xi}-2\left(3 \Psi_{0}^{2} \Psi_{1}\right)_{\xi \xi \xi}-2\left(3 \Psi_{0}^{2} \Psi_{1}\right)_{\xi \zeta \zeta}\right]\right], \\
= & \frac{3}{32} \eta^{5} \sinh \left[\frac{1}{6}(\xi+\zeta)\right]\left[765 \cosh ^{4}\left[\frac{1}{6}(\xi+\zeta)\right]-729 \cosh ^{2}\left[\frac{1}{6}(\xi+\zeta)\right]+91\right] \frac{\mathfrak{J}^{2 \rho}}{\Gamma(2 \rho+1)}, \\
\Psi_{3}(\xi, \zeta, \mathfrak{J})= & E^{-1}\left[s^{\rho} E\left[-\left(3 \Psi_{0}^{2} \Psi_{2}+3 \Psi_{0}^{2} \Psi_{1}^{2}\right)_{\xi}-2\left(3 \Psi_{0}^{2} \Psi_{2}+3 \Psi_{0}^{2} \Psi_{1}^{2}\right)_{\xi \xi \xi}-2\left(3 \Psi_{0}^{2} \Psi_{2}+3 \Psi_{0}^{2} \Psi_{1}^{2}\right)_{\xi \zeta \zeta}\right]\right], \\
= & -\frac{3}{128} \cosh \left[\frac{1}{6}(\xi+\zeta)\right]\left[171738 \cosh ^{6}\left[\frac{1}{6}(\xi+\zeta)\right]-349884 \cosh ^{4}\left[\frac{1}{6}(\xi+\zeta)\right]+215496 \cosh ^{2}\left[\frac{1}{6}(\xi+\zeta)\right]\right. \\
& -36907] \frac{\mathfrak{J}^{2 \rho}}{\Gamma(2 \rho+1)} .
\end{aligned}
$$




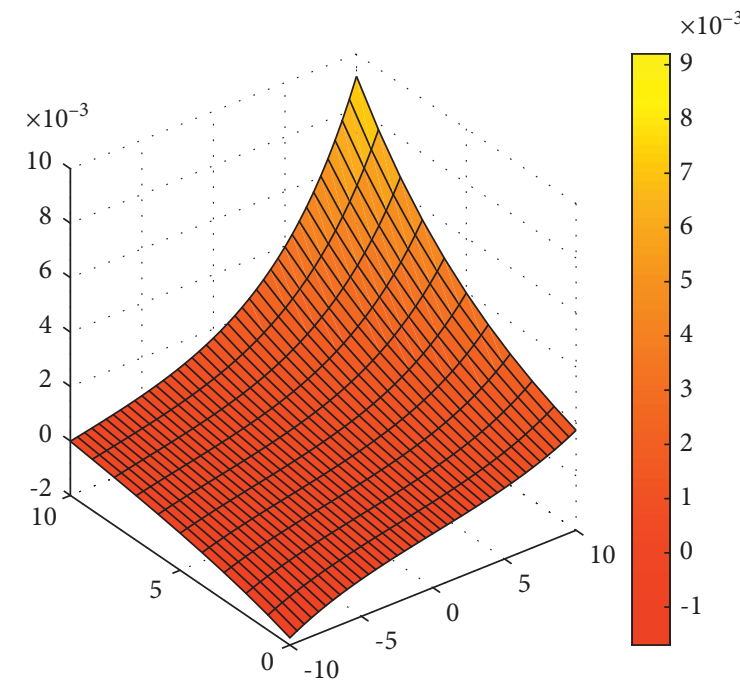

(a)

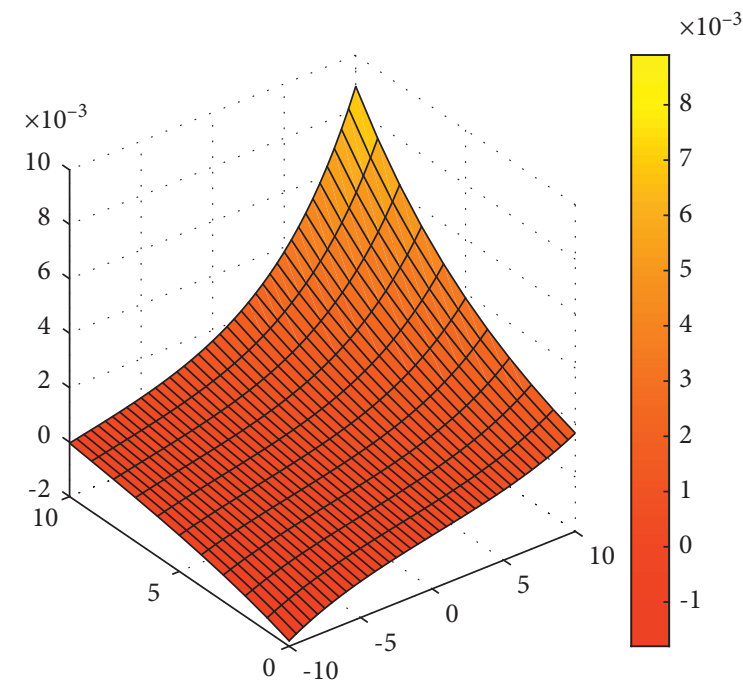

(c)

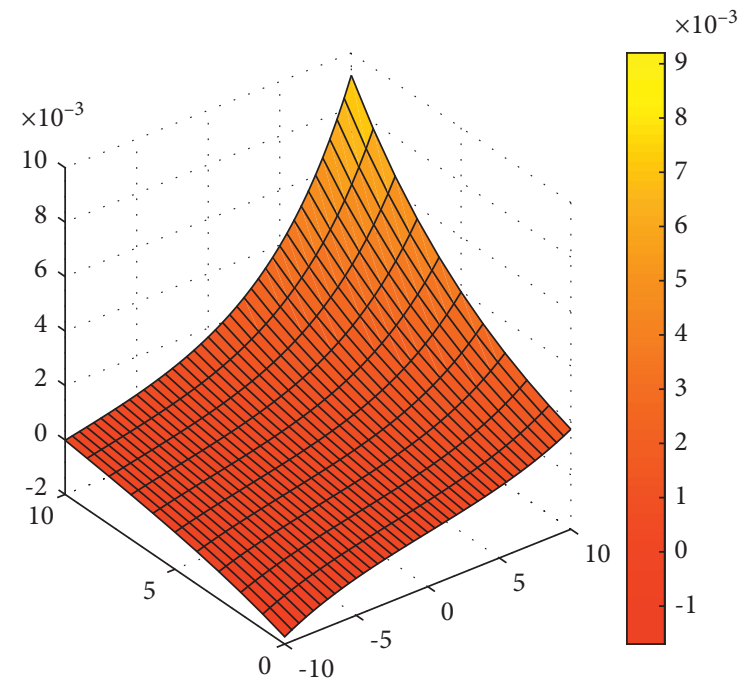

(b)

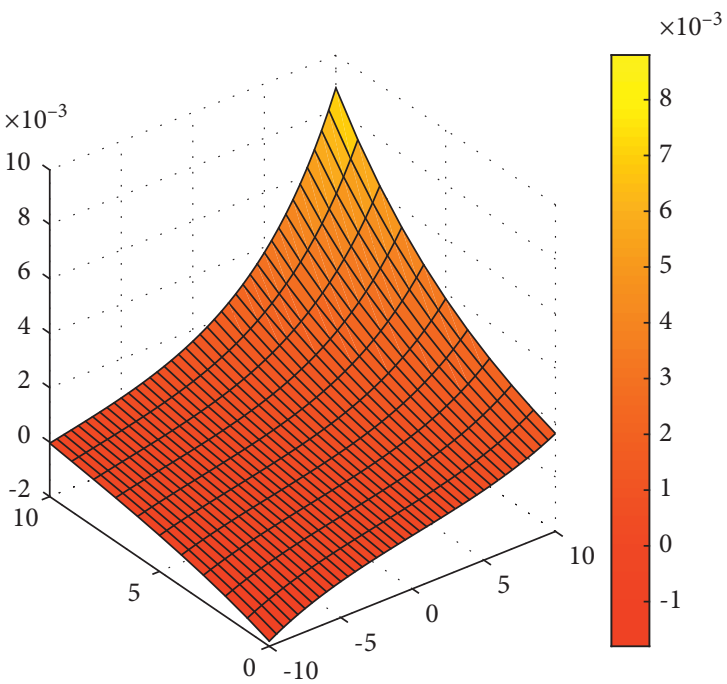

(d)

Figure 3: (a) The exact solution figure of $\Psi(\xi, \mathfrak{J})$ of Example 2. (b) The EDM solution figure of $\Psi(\xi$, $)$ of Example 2. (c) The EDM solution figure of $\Psi(\xi, \mathfrak{I})$ at $\rho=0.8$ Example 2. (d) The EDM solution figure of $\Psi(\xi, \mathfrak{I})$ at $\rho=0.6$ Example 2. 


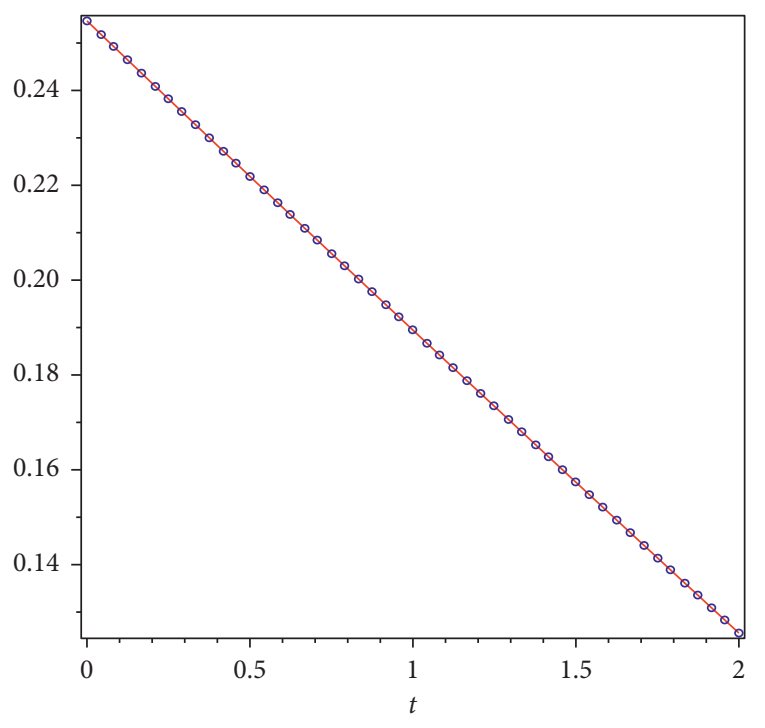

(a)

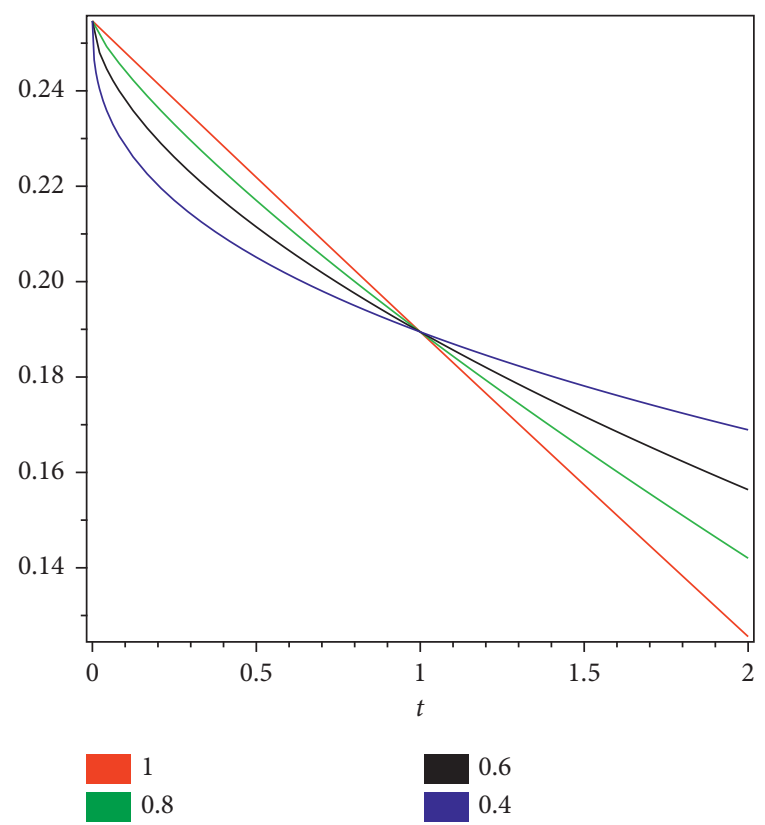

(b)

FIgURE 4: (a) The exact and EDM solution of $\Psi(\xi, \mathfrak{\Im})$ with respect to $\mathfrak{~ E x a m p l e ~ 1 . ~ ( b ) ~ T h e ~ E D M ~ s o l u t i o n ~ f i g u r e ~ o f ~ d i f f e r e n t ~ f r a c t i o n a l - o r d e r ~}$ of $\rho$ with respect to $\mathfrak{I}$ of Example 1 .

The EDM result is

$$
\begin{aligned}
\Psi(\xi, \zeta, \mathfrak{I})= & \Psi_{0}(\xi, \zeta, \mathfrak{I})+\Psi_{1}(\xi, \zeta, \mathfrak{I})+\Psi_{2}(\xi, \zeta, \mathfrak{I})+\Psi_{3}(\xi, \zeta, \mathfrak{J})+\cdots \\
\Psi(\xi, \zeta, \mathfrak{I})= & \frac{3}{2} \eta \sinh \left[\frac{1}{6}(\xi+\zeta)\right]-\left[3 \eta^{3} \sinh ^{2}\left[\frac{1}{6}(\xi+\zeta)\right] \cosh \left[\frac{1}{6}(\xi+\zeta)\right]\right. \\
& \left.+\frac{3}{8} \eta^{3} \cosh ^{3}\left[\frac{1}{6}(\xi+\zeta)\right]\right] \frac{\mathfrak{J}^{\rho}}{\Gamma(\rho+1)}+\frac{3}{32} \eta^{5} \sinh \left[\frac{1}{6}(\xi+\zeta)\right] \\
& {\left[765 \cosh ^{4}\left[\frac{1}{6}(\xi+\zeta)\right]-729 \cosh ^{2}\left[\frac{1}{6}(\xi+\zeta)\right]+91\right] \frac{\mathfrak{J}^{2 \rho}}{\Gamma(2 \rho+1)} } \\
& -\frac{3}{128} \cosh ^{2 \rho}\left[\frac{1}{6}(\xi+\zeta)\right]\left[171738 \cosh ^{6}\left[\frac{1}{6}(\xi+\zeta)\right]-349884\right. \\
& \left.\cosh ^{4}\left[\frac{1}{6}(\xi+\zeta)\right]+215496 \cosh ^{2}\left[\frac{1}{6}(\xi+\zeta)\right]-36907\right] \frac{\mathfrak{J}^{2 \rho}}{\Gamma(2 \rho+1)}+\cdots
\end{aligned}
$$

For $\rho=1$,

$$
\Psi(\xi, \mathfrak{\Im})=\frac{3}{2} \eta \sinh \left[\frac{1}{6}(\xi+\zeta-\eta \Im)\right] .
$$

In Figure 3, the exact and the EDM solutions of Example 2 at $\rho=1$ are shown by Figures 3(a) and 3(b), respectively. From the given figures, it can be seen that both the EDM and exact solutions are in close contact with each other. Also, in Figures 3(c) and 3(d), the EDM results of problem 2 are investigated at different fractional-order $\rho=0.8$ and 0.6. It is analyzed that time-fractional problem results are convergent to an integer order effect as time-fractional analysis to integer order. In Figure 4, the first graph shows the two dimensions of exact and analytical solutions with respect to $\mathfrak{I}$ and the second one shows the different fractional-order graph with respect to $\mathfrak{J}$.

\section{Conclusion}

In this article, we investigated the time-fractional Zakharov-Kuznetsov equations using an Elzaki decomposition method. The given test examples illustrate the leverage and 
effectiveness of the suggested method. The obtained solutions are demonstrated by tables and graphs. The Elzaki decomposition method solution is in close contact with the actual result of the given problems. The figures show that the time-fractional solutions obtained have verified the convergence towards the integer order solutions. Moreover, the current technique is simple and straightforward as compared to other analytical techniques; the proposed method can solve other linear and nonlinear fractional-order partial differential equations.

\section{Data Availability}

The numerical data used to support the findings of this study are included within the article.

\section{Disclosure}

Pongsakorn Sunthrayuth and Farman Ali are the co-first authors.

\section{Conflicts of Interest}

The authors declare that there are no conflicts of interest regarding the publication of this article.

\section{Authors' Contributions}

Pongsakorn Sunthrayuth and Farman Ali contributed equally to this work.

\section{Acknowledgments}

The authors extend their appreciation to the Deanship of Scientific Research at King Khalid University, Saudi Arabia, for funding this work through Research Groups Program under grant number (R.G.P2./99/41).

\section{References}

[1] A. Ekinci and M. Ozdemir, "Some new integral inequalities via riemann-liouville integral operators," Applied and Computational Mathematics, vol. 18, 2019.

[2] Q. Li, Z. Zheng, S. Wang, and J. Liu, Lattice Boltzmann Model for Nonlinear Heat Equations, Springer, Berlin, Germany, 2012.

[3] H. Khan, R. Shah, M. Arif, and S. Bushnaq, "The Chebyshev wavelet method (CWM) for the numerical solution of fractional HIV infection of CD T cells model," International Journal of Algorithms, Computing and Mathematics, vol. 6, no. 2, pp. 1-17, 2020.

[4] J. Nathan Kutz, J. L. Proctor, and S. L. Brunton, "Applied Koopman theory for partial differential equations and datadriven modeling of spatio-temporal systems," Complexity, vol. 2018, Article ID 6010634, 16 pages, 2018.

[5] V. E. Zakharov and E. A. Kuznetsov, "Three-dimensional solitons," Soviet Physics Uspekhi, vol. 39, pp. 285-286, 1974.

[6] A.-M. Wazwaz, "The extended tanh method for the ZakharovKuznetsov (ZK) equation, the modified ZK equation, and its generalized forms," Communications in Nonlinear Science and Numerical Simulation, vol. 13, no. 6, pp. 1039-1047, 2008.
[7] A. Biswas and E. Zerrad, "Solitary wave solution of the Zakharov-Kuznetsov equation in plasmas with power law nonlinearity," Nonlinear Analysis: Real World Applications, vol. 11, no. 4, pp. 3272-3274, 2010.

[8] H.-L. Zhen, B. Tian, H. Zhong, and Y. Jiang, "Dynamic behaviors and soliton solutions of the modified ZakharovKuznetsov equation in the electrical transmission line," Computers \& Mathematics with Applications, vol. 68, no. 5, pp. 579-588, 2014.

[9] A. R. Seadawy, "Stability analysis for two-dimensional ionacoustic waves in quantum plasmas," Physics of Plasmas, vol. 21, no. 5, Article ID 52107, 2014.

[10] A. H. Khater, D. K. Callebaut, W. Malfliet, and A. R. Seadawy, "Nonlinear dispersive Rayleigh-taylor instabilities in magnetohydrodynamic flows," Physica Scripta, vol. 64, no. 6, pp. 533-547, 2001.

[11] A. H. Khater, D. K. Callebaut, and A. R. Seadawy, "Nonlinear dispersive instabilities in kelvin-helmholtz magnetohydrodynamic flows," Physica Scripta, vol. 67, no. 4, pp. 340-349, 2003.

[12] A. R. Seadawy, "Fractional solitary wave solutions of the nonlinear higher-order extended $\mathrm{KdV}$ equation in a stratified shear flow: Part I," Computers \& Mathematics with Applications, vol. 70, no. 4, pp. 345-352, 2015.

[13] S. S. Ray and S. Sahoo, "New exact solutions of fractional zakharov-kuznetsov and modified zakharov-kuznetsov equations using fractional sub-equation method," Communications in Theoretical Physics, vol. 63, no. 1, pp. 25-30, 2015.

[14] A. Prakash, M. Kumar, and D. Baleanu, "A new iterative technique for a fractional model of nonlinear ZakharovKuznetsov equations via Sumudu transform," Applied Mathematics and Computation, vol. 334, pp. 30-40, 2018.

[15] D. Kumar, J. Singh, and S. Kumar, "Numerical computation of nonlinear fractional Zakharov-Kuznetsov equation arising in ion-acoustic waves," Journal of the Egyptian Mathematical Society, vol. 22, no. 3, pp. 373-378, 2014.

[16] A. R. Seadawy, "Three-dimensional nonlinear modified Zakharov-Kuznetsov equation of ion-acoustic waves in a magnetized plasma," Computers \& Mathematics with Applications, vol. 71, no. 1, pp. 201-212, 2016.

[17] P. Veeresha and D. G. Prakasha, "Solution for fractional Zakharov-Kuznetsov equations by using two reliable techniques," Chinese Journal of Physics, vol. 60, pp. 313-330, 2019.

[18] M. G. Saker, F. Erdogan, and A. Yildirim, "Variational iteration method for the time fractional Fornberg-Whitham equation," Computers \& Mathematics with Applications, vol. 63, no. 9, pp. 1382-1388, 2012.

[19] K. S. Miller and B. Ross, An Introduction to the Fractional Calculus and Fractional Differential Equations, Wiley, New York, NY, USA, 1993.

[20] A. A. Kilbas, H. M. Srivastava, and J. J. Trujillo, Theory and Applications of Fractional Differential Equations, Elsevier, Amsterdam, Netherland, 2006.

[21] H. Khan, R. Shah, P. Kumam, B. Dumitru, and M. Arif, "Laplace decomposition for solving nonlinear system of fractional order partial differential equations," Advances in Difference Equations, vol. 1, pp. 1-18, 2020.

[22] M. A. E. Herzallah, M. A. El-Sayed, and D. Baleanu, "On the fractional-order diffusion-wave process," Romanian Journal of Physics, vol. 55, no. 3-4, pp. 274-284, 2010.

[23] D. Baleanu, O. Defterli, and O. P. Agrawal, "A central difference numerical scheme for fractional optimal control problems," Journal of Vibration and Control, vol. 15, no. 4, pp. 583-597, 2009. 
[24] A. M. Wazwaz, "A reliable technique for solving linear and nonlinear Schrodinger equations by Adomian decomposition method," Bulletin of institute of mathematics, vol. 29, no. 2, pp. 125-134, 2001.

[25] A.-M. Wazwaz, "A study on linear and nonlinear Schrodinger equations by the variational iteration method," Chaos, Solitons \& Fractals, vol. 37, no. 4, pp. 1136-1142, 2008.

[26] R. Shah, H. Khan, D. Baleanu, P. Kumam, and M. Arif, “The analytical investigation of time-fractional multi-dimensional Navier-Stokes equation," Alexandria Engineering Journal, vol. 59, no. 5, pp. 2941-2956, 2020.

[27] T. M. Elzaki, "The new integral transform "Elzaki Transfrom"," Global Journal of Pure and Applied Mathematics, vol. 7, no. 1, pp. 57-64, 2011.

[28] G. Adomian, "Solution of physical problems by decomposition," Computers \& Mathematics with Applications, vol. 27, no. 9-10, pp. 145-154, 1994.

[29] G. Adomian, "A review of the decomposition method in applied mathematics," Journal of Mathematical Analysis and Applications, vol. 135, no. 2, pp. 501-544, 1988.

[30] E. Aksoy, A. C. Çevikel, and A. Bekir, "Soliton solutions of (2+1)-dimensional time-fractional zoomeron equation," Optik, vol. 127, no. 17, pp. 6933-6942, 2016.

[31] R. I. Nuruddeen, "Elzaki decomposition method and its applications in solving linear and nonlinear Schrodinger equations," Sohag Journal of Mathematics, vol. 4, no. 2, pp. 1-5, 2017.

[32] A. Akbulut and M. Kaplan, "Auxiliary equation method for time-fractional differential equations with conformable derivative," Computers \& Mathematics with Applications, vol. 75, no. 3, pp. 876-882, 2018.

[33] K. Hosseini, A. Korkmaz, A. Bekir, F. Samadani, A. Zabihi, and M. Topsakal, "New wave form solutions of nonlinear conformable time-fractional zoomeron equation in $(2+1)$ dimensions," Wave Random Complex, vol. 31, 2019.

[34] D. Kumar and M. Kaplan, "New analytical solutions of $(2+$ 1)-dimensional conformable time fractional Zoomeron equation via two distinct techniques," Chinese Journal of Physics, vol. 56, pp. 2173-2185, 2018.

[35] H. K. Mishra and R. K. Pandey, "Time-fractional nonlinear dispersive type of the Zakharov- Kuznetsov equation via HAFSTM," Proceedings of the National Academy of Sciences, India Section A: Physical Sciences, vol. 91, pp. 1-14, 2020.

[36] M. Odabasi, "Traveling wave solutions of conformable timefractional Zakharov-Kuznetsov and Zoomeron equations," Chinese Journal of Physics, vol. 64, pp. 194-202, 2020. 